\title{
Modeling of absorption process of the water by the wood in two-dimensions
}

\author{
Nadia Amardo ${ }^{1}$, Fatima Ouzidan ${ }^{1}$, Rachid Atmani ${ }^{1}$, Faiçal Moubarak ${ }^{1}$, M'hammed El Kouali ${ }^{1}$, \\ Mohammed Talbi ${ }^{1}$, Abdalhak El Brouzi ${ }^{2}$ \\ ${ }^{1}$ Laboratory of Analytical Chemistry and Physical Chemistry of Materials Sciences Faculty Ben M'sik, Hassan \\ II University of Casablanca; Morocco \\ ${ }^{2}$ Laboratory of Physical Chemistry and Electrochemistry Faculty of Science and Technology Mohammedia. \\ Hassan II University of Casablanca; Morocco
}

\begin{abstract}
Wood is a natural and highly anisotropic material. Therefore, mechanical characteristics of the material depend on the direction of wood. In this paper, we propose to describe the transfer of the water inside the wood in two dimensions by immersing the sample in liquid water. The potential which drives the transport of water through the wood is controlled by the diffusion transient process. A model based on a numerical finite difference method has been found to follow the absorption process of water above the fibers saturation point of two-dimensions.
\end{abstract}

Keywords: Wood, Transfer, Absorption, modeling, finite difference method.

\section{Introduction}

Wood is probably the first material that has been used by man. Its use has constantly developed, but its limitations are well known. These include, for example, its dimensional instability or biodegradation. Wood is a hygroscopic material whose role has always been crucial in the history of mankind. The development of technology today makes it possible to study the physical and chemical properties of wood, whose water status depends on the climatic conditions of humidity and temperature of the environment. It contains the water in two forms. When the moisture content is at its highest, there is absorbed water inside the cell walls and free water contained in the voids inside the cells ${ }^{1,2}$. The fiber saturation point (FSP) is the moisture content obtained when the wood dries, where only the water absorbed inside the walls remains. The free water inside the cell cavities having evaporated first, during drying $^{3,4}$.

Wood is an anisotropic material with three main diffusion axes and three principal diffusivities, the longitudinal diffusivity being more than ten times greater than the radial and tangential diffusivities which are approximately equal ${ }^{5,6}$. Although, the main diffusivities are concentration-dependent in the case of moisture transport below the saturation point of the fibers (FSP), they can be considered constant when the moisture content is beyond FSP. This is the case when the wood is immersed in water; it is obvious that

*Corresponding author: Nadia Amardo

Email address: n.amardo@gmail.com

DOI: http://dx.doi.org/10.13171/mjc9602001181125na its water concentration is higher than that associated with the fibre saturation point. This water absorption is an important phenomenon because it can release the chemical previously located in the wood for its protection. On the other hand, this water transport can be used to control the release of iodine or other materials into the water. Polymers have already been successfully used to control chemical releases to water, and applications have been found in agriculture. While the problem of releasing iodine into the water at a controlled rate is a problem in undeveloped countries where people suffer from goitrous diseases, attempts have been made using silicone devices. Silicone devices are relatively complex and expensive, so wood can be used to control the rate of iodine release into water.

The transfer of the water inside the wood is governed by the Fickian diffusion, the strength transfers of driving being the gradient of concentration and the second law of Fick can express the change of concentration with time.

The main purpose of this study is to describe the absorption process of water by the wood in twodimensions, when the water content is above the fiber saturation point ${ }^{7}$.

The second aim of this work is to build and check a numerical model able to describe the absorption process in two dimensions, when the water content is above the fiber saturation point. Some models have been successfully applied in cases where the water 
content is above the fiber saturation point in onedimensional transfer ${ }^{8-12}$.

\section{Theoretical approach}

The study of water diffusion inside the wood is based on the following assumptions:

1- The square section of a beam is considered, with a two-dimensional transfer.

2- The transfer of water is controlled by the transient diffusion through the wood.

3- The diffusivity for the two dimensions remains constant during the experiment.

4- The diffusion coefficient is independent of the concentration.

5- As soon as the sample is brought into contact with the water, the water content on the wood surfaces reach equilibrium.

$$
\begin{array}{r}
\frac{C-C_{e}}{C_{0}-C_{e}}=\frac{16}{\pi^{2}}\left(\sum_{n=0}^{\infty} \frac{1}{2 n+1} \cdot \sin \frac{(2 n+1) \pi x}{X} \cdot \exp \left(\frac{(2 n+1)^{2} \pi^{2} D t}{-X^{2}}\right)\right) \\
\cdot\left(\sum_{m=0}^{\infty} \frac{1}{2 m+1} \cdot \sin \frac{(2 m+1) \pi y}{Y} \exp \left(\frac{(2 m+1)^{2} \pi^{2} D t}{-Y^{2}}\right)\right)
\end{array}
$$

\subsection{Numerical analysis}

The diffusion method will be simulated from an algorithm using a numerical method of explicit twodimensional finite differences along the cross-section perpendicular to the faces of the sample ${ }^{13,14}$.

\subsection{Analytical analysis}

The transfer of water through the wood is governed by transient diffusion in two-dimensions:

$\frac{\partial \mathrm{C}}{\partial \mathrm{t}}=\mathrm{D}_{\mathrm{X}} \frac{\partial^{2} \mathrm{C}}{\partial \mathrm{X}^{2}}+\mathrm{D}_{\mathrm{Y}} \frac{\partial^{2} \mathrm{C}}{\partial \mathrm{Y}^{2}}$

- $\quad \mathrm{D}_{\mathrm{X}}$ and $\mathrm{D}_{\mathrm{Y}}$ are the main diffusivities according to the directions of the wood used.

- $\quad \mathrm{C}$ is the water content.

- The limit condition is: $\mathrm{C}=$ Constant $=C e$

- $\quad C e$ is the concentration at balance.

The initial concentration, $\mathrm{C}_{\mathrm{o}}$ is uniform. In this case, the analytical solution can be used for $0<\mathrm{x}<\mathrm{X}$; $0<\mathrm{y}$ $<\mathrm{Y}$, given by:

$$
\left\{\begin{array}{ccc}
\text { x }=\text { i. } \Delta X & 0 \leq \mathrm{i} \leq 2 \mathrm{~N} & \mathrm{X}=2 \mathrm{~N} . \Delta \mathrm{X} \\
\mathrm{y}=\mathrm{j} . \Delta \mathrm{Y} & 0 \leq \mathrm{j} \leq 2 \mathrm{~N} & \mathrm{Y}=2 \mathrm{~N} . \Delta \mathrm{Y}
\end{array}\right.
$$

The matter equilibrium is evaluated during the increment of time inside the cube of thickness $\Delta_{\mathrm{X}}$ or $\Delta_{\mathrm{Y}}$ centered at the position $\mathrm{i}$ and $\mathrm{j}$ (Figure 1), considering the radial diffusion, tangential and longitudinal of the wood on both sides ${ }^{15-17}$.
For this purpose the water concentration profile in the wood is discredited in a number of $2 \mathrm{~N}$ equal slices of thickness $\mathrm{X}$ and $\mathrm{Y}$ and diffusion is carried out over small intervals of time $\Delta t$. Each position obtained is defined by two integers $\mathrm{i}$ and $\mathrm{j}$, so that:

$$
\begin{gathered}
\mathrm{CN}_{\mathrm{i}, \mathrm{j}}=\mathrm{C}_{\mathrm{i}, \mathrm{j}}+\frac{1}{\mathrm{M}_{\mathrm{X}}}\left[\mathrm{C}_{\mathrm{i}+1, \mathrm{j}}-2 \mathrm{C}_{\mathrm{i}, \mathrm{j}}+\mathrm{C}_{\mathrm{i}-1, \mathrm{j}}\right]+\frac{1}{\mathrm{M}_{\mathrm{Y}}}\left[\mathrm{C}_{\mathrm{i}, \mathrm{j}+1}-2 \mathrm{C}_{\mathrm{i}, \mathrm{j}}+\mathrm{C}_{\mathrm{i}, \mathrm{j}-1}\right] \\
\text { With: } \quad \mathrm{M}_{\mathrm{X}}=\frac{(\Delta \mathrm{X})^{2}}{\mathrm{D}_{\mathrm{X}} \cdot \Delta \mathrm{t}} \quad \text { and } \quad M_{Y}=\frac{(\Delta \mathrm{Y})^{2}}{\mathrm{D}_{\mathrm{Y}} \cdot \Delta \mathrm{t}}
\end{gathered}
$$

The new concentration after the lapse of time at the position i, j, $C N_{i, j}$ can therefore be expressed in terms of the internal concentrations obtained at the same place and the adjacent places:

This method leads to:

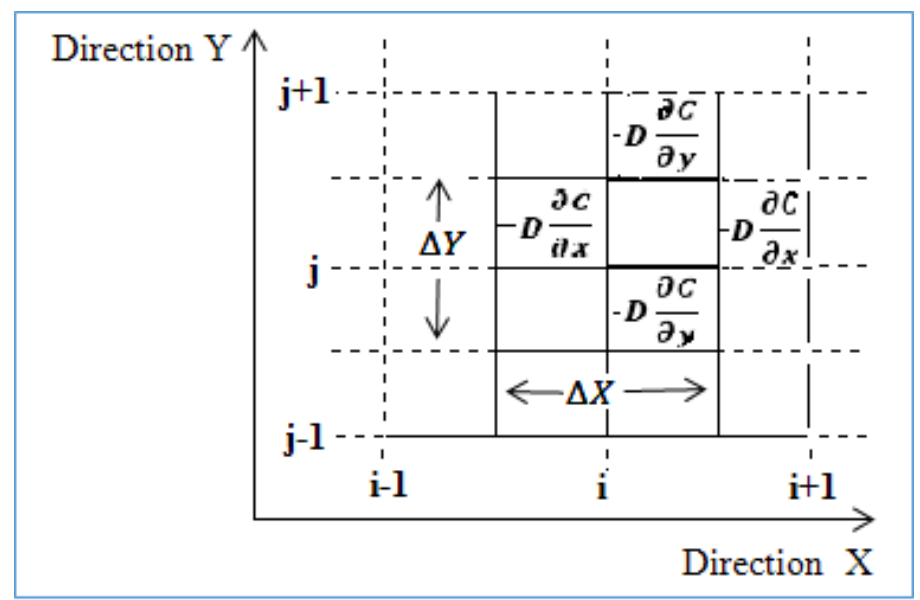

Figure 1. Space-time diagram of two transfer dimensions inside the sample 


\section{Experimental procedure}

\subsection{Material used}

Several samples of Moroccan beech wood of cubic shape are used in the study. They are cut in such a way that their three axes are the same, are the three main axes of diffusion.

\subsection{Procedure adopted}

Indeed, the cubic shape samples of $2 \mathrm{~cm}$ side were cut in the radial, tangential and longitudinal directions of the wood and the diffusion through these four opposite faces is only obtained by protecting the two other faces with the using waterproof film. The beech wood samples were immersed in a beaker of capacity $250 \mathrm{ml}$ contained water; the absorption kinetic has been determined by weighing. The time interval was selected until having a constant weight gain.
We first calculated the diffusivities of each direction of wood, namely longitudinal, radial and tangential, by considering only the one-dimensional transfer through the wood, while protecting the other four faces with an impermeable film. As well as the principal diffusivity is calculated from the line obtained by tracing the amount $\left(Q_{t}\right)$ of water transported by the corresponding axis as a function of the square root of time, the diffusivity is easily calculated when reached at equilibrium $\left(Q_{\infty}\right)$, but in the two-dimensional transfer, only two diffusivities were used to validate the experimental data.

$\frac{\mathrm{Q}_{\mathrm{t}}}{\mathrm{Q}_{\infty}}=\frac{4}{\mathrm{~L}}\left(\frac{\mathrm{Dt}}{\pi}\right)^{0,5} \quad 18-22$

Table 1. The diffusivity of each direction.

\begin{tabular}{|l|l|}
\hline \multicolumn{1}{|c|}{ Main axes } & \multicolumn{1}{c|}{ Diffusivity $\left(\mathrm{cm}^{2} / \mathbf{s}\right)$} \\
\hline Longitudinal & $\mathrm{D}_{\mathrm{L}}=2.016 \times 10^{-6}$ \\
\hline Tangential & $\mathrm{D}_{\mathrm{T}}=1.7 \times 10^{-6}$ \\
\hline Radial & $\mathrm{D}_{\mathrm{R}}=1.7 \times 10^{-6}$ \\
\hline
\end{tabular}

\section{Results and discussion}

\subsection{Kinetics of water absorption}

Figure 2 describes water absorption as a function of immersion time and shows the evolution of water absorbed by the wood in the longitudinal-tangential, longitudinal-radial and radial-tangential directions. As expected, it shows that water absorption is faster in the first stage of the absorption process in the longitudinal-tangential direction (a). In addition, radial diffusion is favored by the orientation of the wood rays (serial or non-serial rays, sometimes consisting only of coated cells). On the one hand, the pores of the cells constituting the woody rays favor the transfer of bound water. In the radial direction and on the other hand, the walls of the cells interfere with the transfer in the tangential direction.

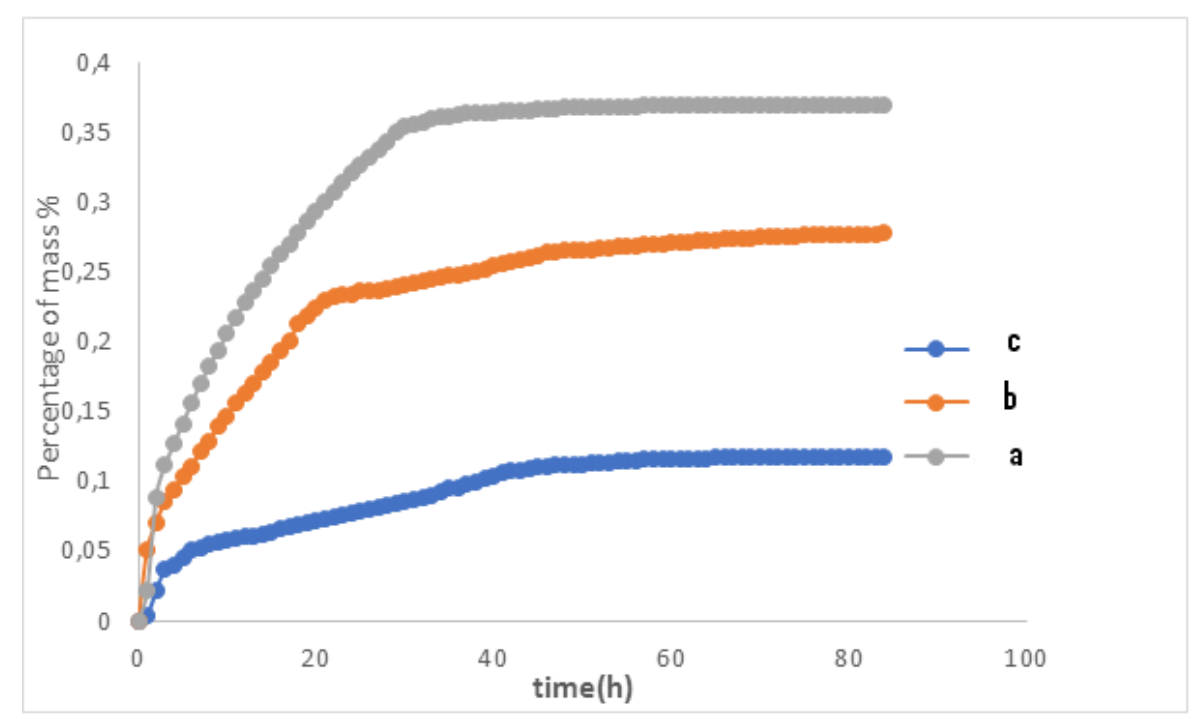

Figure 2. Absorption kinetics of water within cubics specimens $(2 \times 2 \times 2 \mathrm{~cm})$ as function the time in 2D direction the wood. (a) longitudinal-tangential, (b) longitudinal-radial, (c) radial-tangential 


\subsection{Concentration profiles}

The profiles of water concentration developed through the thickness of the sample $2 \mathrm{~cm}$ in $2 \mathrm{D}$ direction can be calculated by using the numerical model of finite differences method, whatever the initial conditions in the case of absorption. These profiles of concentration provide a deeper insight into the water distribution and migration of water in wood in terms of water concentration. Figures 3, 4 and 5 give profiles of the water content represented by the vertical axis and the horizontal plane represents the two directions of transfer.

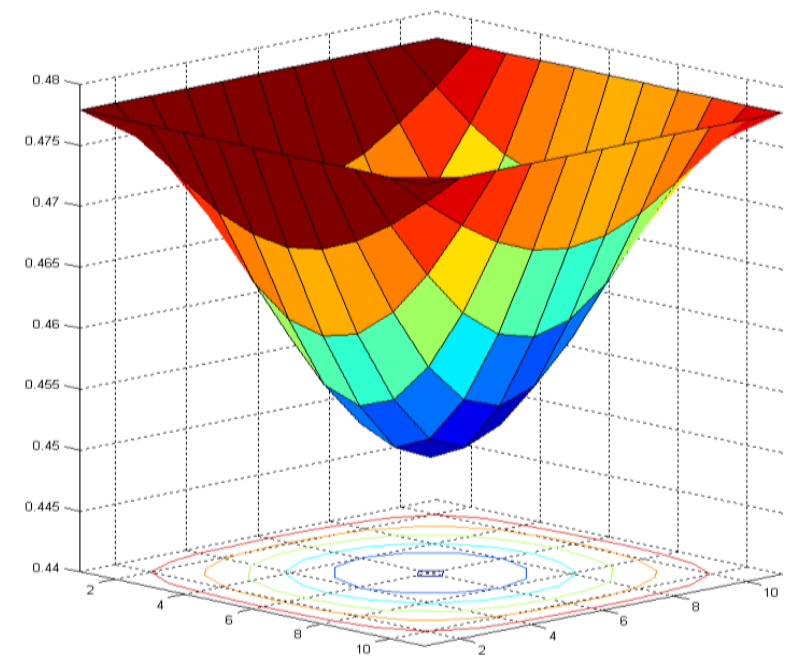

Figure 3. Concentration profile in the two-dimensional direction (longitudinal and radial).

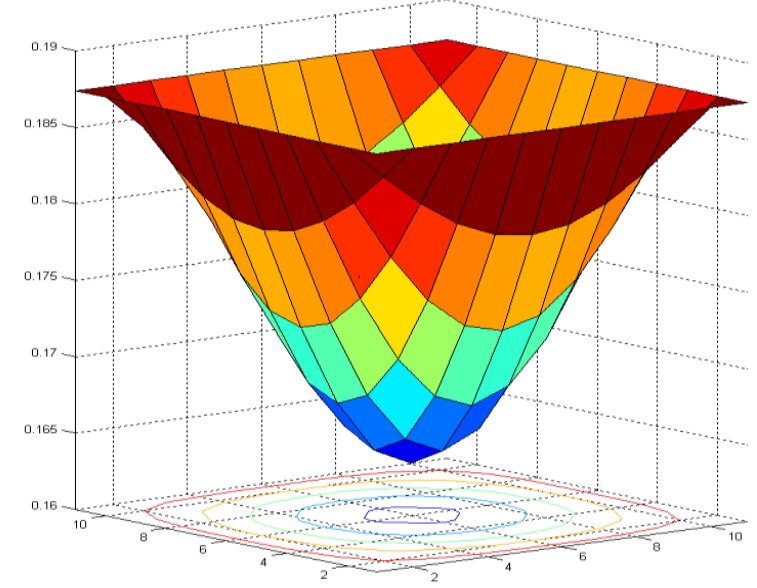

Figure 4. Concentration profile in the two-dimensional direction (radial and tangential).

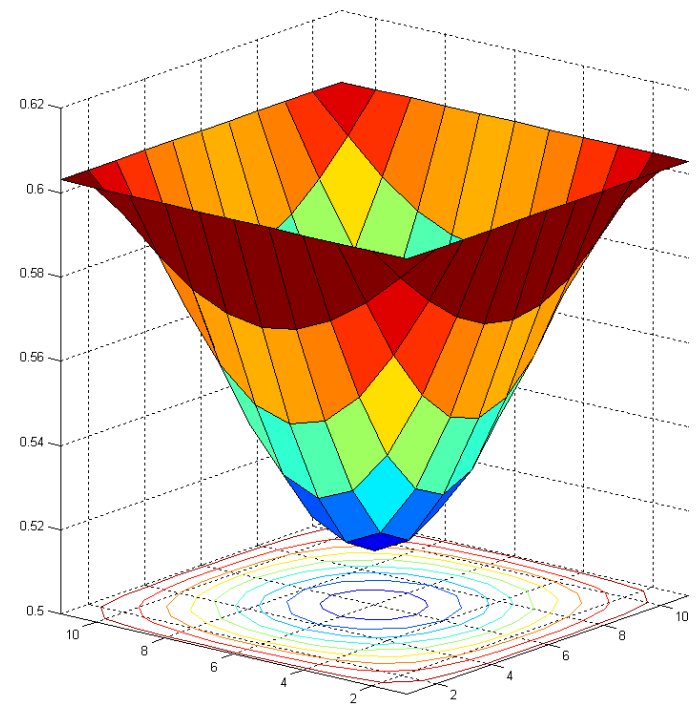

Figure 5. Concentration profile in the two-dimensional direction (longitudinal and tangential). 
These figures show the distribution of water through the wood along the longitudinal-tangential, longitudinal-radial and radial-tangential directions. The color scale of concentration of the water was chosen to obtain a better contrast within wood. The colors ranged from brown, indicating the highest concentration of water, to dark blue, indicating zero concentration. Moreover, the profiles can illustrate the absorption process very easily, when the water content is higher than the fiber saturation point. These profiles are symmetrical with respect to the bisector of the angle formed by the two sides ox and oy, for two reasons; the section perpendicular to the sample faces of the beam is square, the diffusivity is the same along the two perpendicular axes of diffusion. The water content reaches the equilibrium value on the surface during the absorption.

\subsection{Model validity}

The amount of water uptake as a function of the simulation time is calculated using the model and diffusivity values. As shown in the Figures 6, 7 and 8, the analytical solution and the numerical model give the same curves.

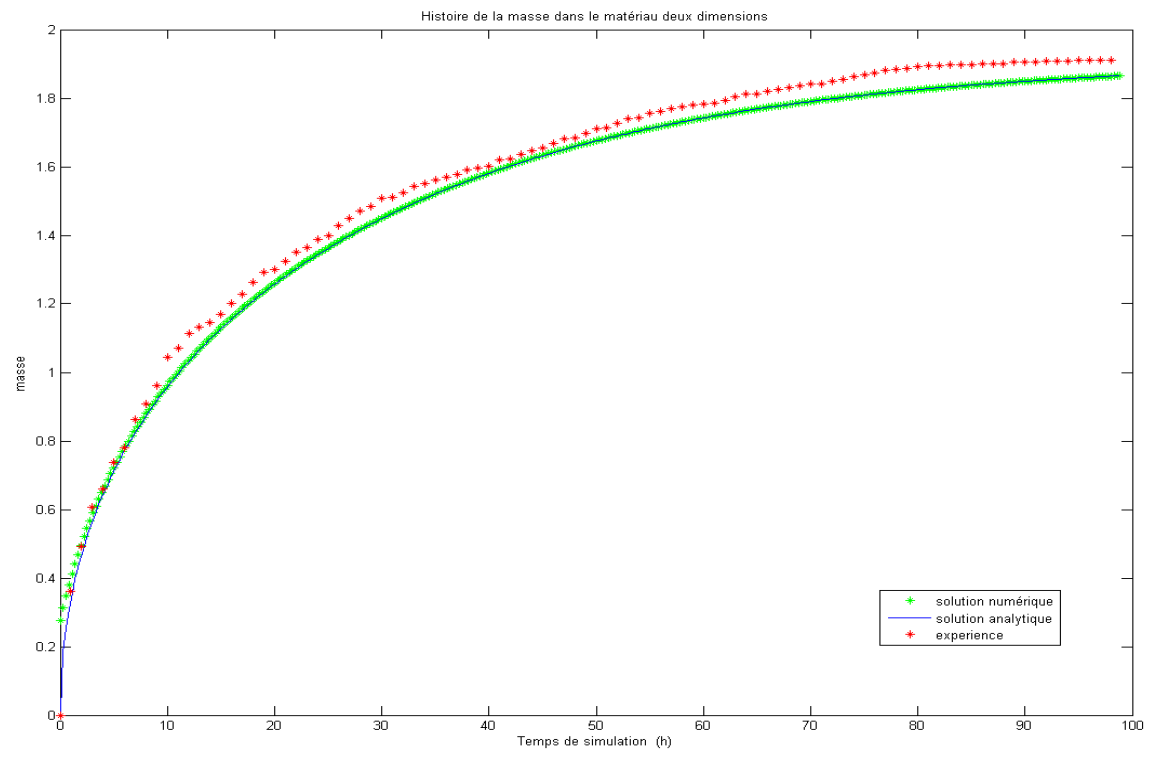

Figure 6. The amount of water transferred to the sample as a function of the simulation time in the twodimensional direction (longitudinal-radial).

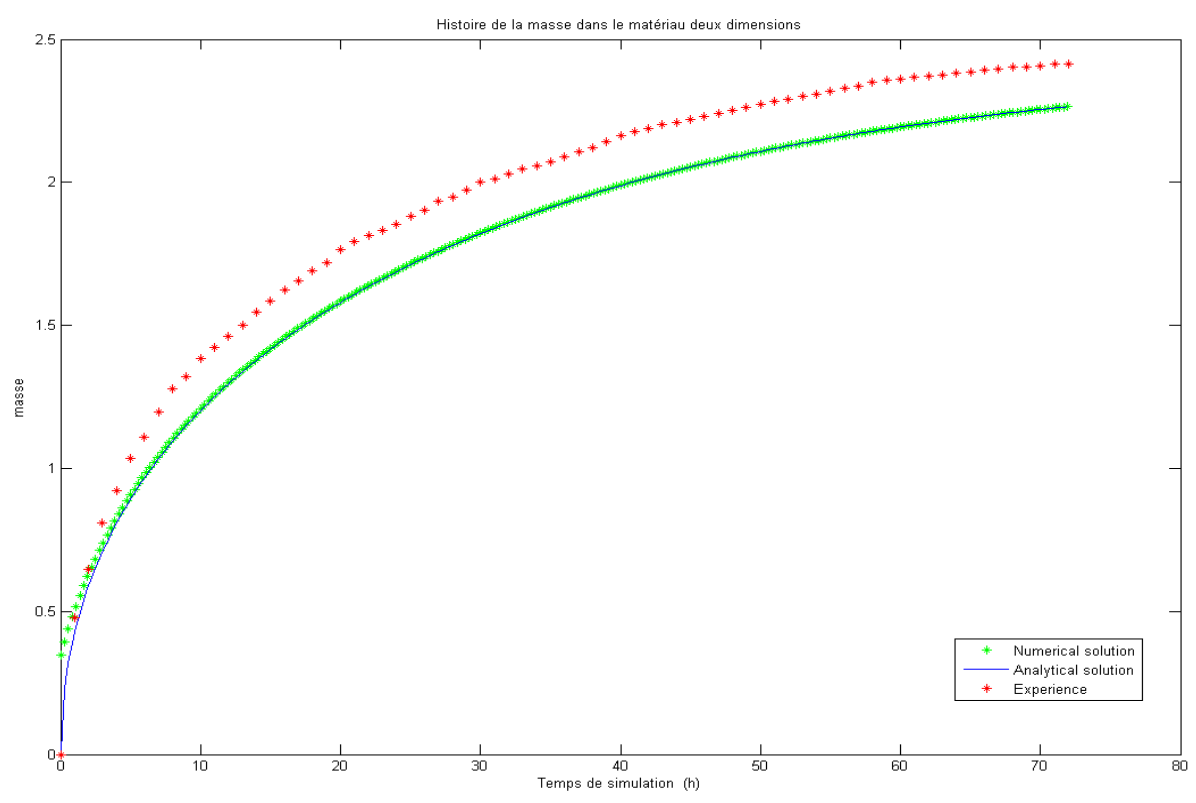

Figure 7. The amount of water transferred to the sample as a function of the simulation time in the twodimensional direction (longitudinal-tangential) 


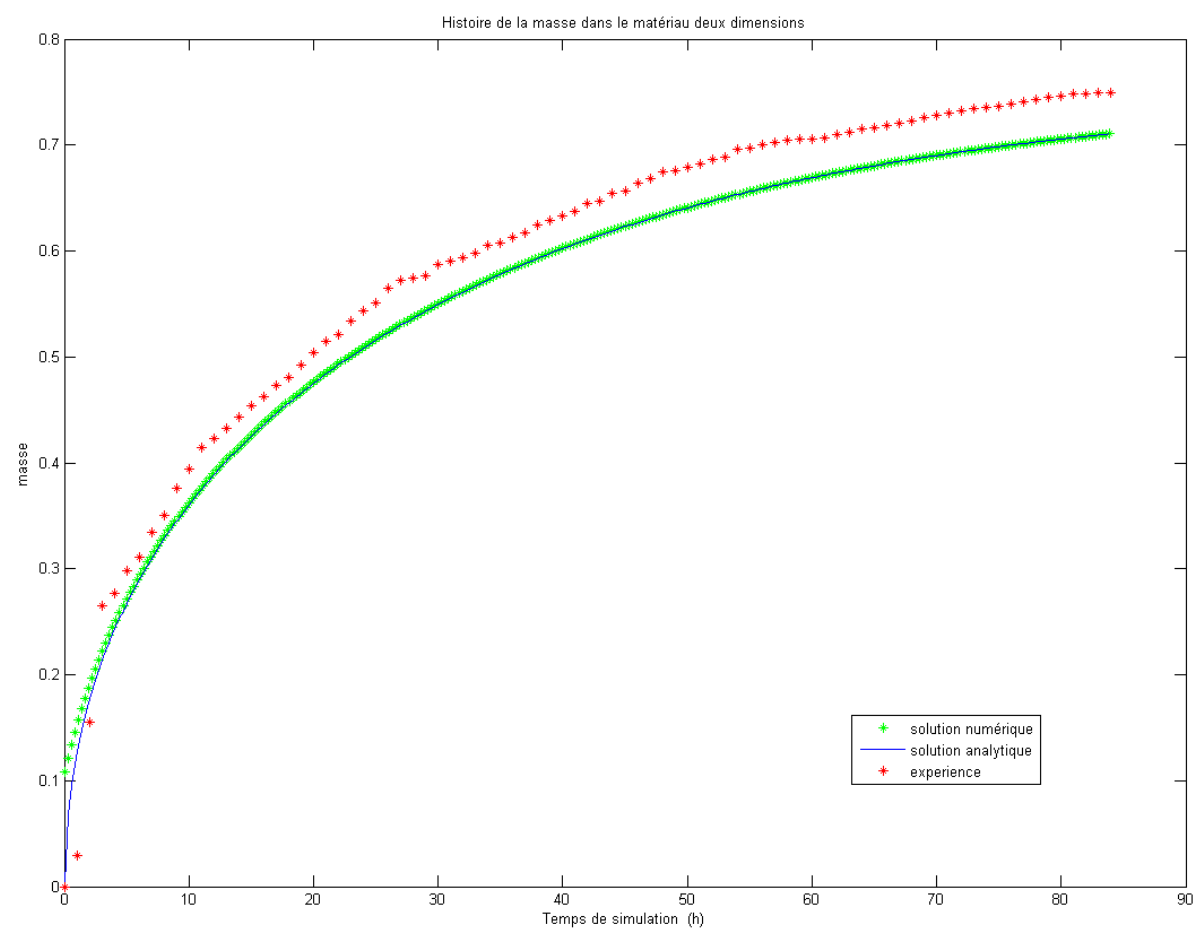

Figure 8. The amount of water transferred to the sample as a function of the simulation time in the twodimensional direction (radial-tangential).

These profiles are calculated in the case of absorption in the two directions (longitudinal-radial, tangentiallongitudinal and radial-tangential). Furthermore, a fairly good agreement is obtained between the experimental results and those calculated by our model. Moreover, the model is able to calculate the water concentration profiles developed across the sample thickness. It is very difficult to determine it by these gradients. However, the proof of their validity exists, because the kinetics of the water absorption is obtained by integrating these concentrations with respect to the thickness of the sample, therefore the model is validated. These concentration profiles are intended to give good information about the water inside wood whose concentration can be easily determined for every point of our sample that is for every point of our sample.

and numerical models can be used in the case of the transfer of a solvent, other than water, into wood, and are capable of determining the solvent content at any time and at any position of the sample.

\section{References}

1- M. Fredriksson, On Wood-Water Interactions in the Over-Hygroscopic Moisture RangeMechanisms, Methods, and Influence of Wood Modification, Forests, 2019, 10, 779.

2- E. E. Thybring, S. V. Glass, S. L. Zelinka, Kinetics of Water Vapor Sorption in Wood Cell Walls: State of the Art and Research Needs, Forests, 2019, 10, 704.

3- H.-S. Yang, H.-J. Kim, H.-J. Park, B.-J. Lee, T.S. Hwang, Water absorption behavior and

\section{Conclusion}

The absorption process of the water by the wood in two dimensions above the fiber saturation point has been studied. Numerical models with finite differences able to describe the absorption process. The model validation has been checked by comparing the theoretical and experimental results. The comparison of the simulations with the experimental results shows the model's ability to give a reliable estimate of the absorption kinetics. The models remain valid for all dimensions, and are able to simulate the process of solvent transfer in wood. The experiments are in agreement with the theoretical results obtained using Fick's second law analytical solutions, under certain initial conditions and at the limits. Other numerical models with finite differences have been developed. These analytical

mechanical properties of lignocellulosic fillerpolyolefin bio-composites, Compos Struct., 2006, 72, 429-437.

4- F. Faure, A. Perrot, S. Pimbert, T. Lecompte, Water absorption measurements on WPCs: Assessment of size and direction dependencies in order to design fast and accurate quality control tests, Polym Test., 2019, 77, 105899.

5- V. V. Rodin, Magnetic Resonance In Studying Natural And Synthetic Materials, Bentham Science Publishers, 2018.

6- P. M. Kekkonen, V.-V. Telkki, J. Jokisaari, Determining the highly anisotropic cell structures of Pinus sylvestris in three orthogonal directions by PGSTE NMR of absorbed water and methane, J Phys Chem B, 2008, 113, 1080-1084. 
7- M. El Kouali, J. Bouzon, J. M. Vergnaud, Modelling the Process of Absorption and Desorption of Water in a Sheet beyond the Fiber Saturation Point by Considering a TwoDimensional Transfer, Holzforschung, 1992, 46, 187-192.

8- N. Amardo, M. E. Kouali, M. Talbi, R. Atmani, F. Moubarak, Z. Mahhate, A. E. Brouzi, Modeling the Absorption of Water by the Wood, International Journal of Science and Research (IJSR), Volume 4 Issue 3, March 2015.

9- H. Mounji, M. El Kouali, J. Vergnaud, Process of absorption of moisture by wood in case of condensation. Modeling and experiment, J Polym Eng., 1993, 12, 197-218.

10- E. Coelho, L. Domingues, J. A. Teixeira, J. M. Oliveira, T. Tavares, Understanding wine sorption by oak wood: Modeling of wine uptake and characterization of volatile compounds retention, Food Res Int., 2019, 116, 249-257.

11- L. Bennani, M. Elkouali, M. Talbi, T. Ainane, Modelling the Absorption Process of Water in Wood in the Transient Regime, International Journal of Chemical Sciences, 2017, 11.

12- E. F. A. Irmak, J. Hanses, S. Schweizer, T. Tröster, A. L. Design, Modeling the Energy Absorption Characteristics of Wood Crash Elements, $12^{\text {th }}$ European LS-DYNA Conference 2019, Koblenz, Germany.

13- R. Atmani, M. E. Kouali, M. Talbi, A. El, H. Argani, Numerical study of absorption and desorption systems of a liquid of simulation by polymer, without evaporation on the surface, Chemical \& Metallurgical Engineering Journal, 2017, 4(1), 1-7.

14- K. Yuniarti, G. Brodie, B. Ozarska, G. Harris, G. Waugh, A mathematical model for moisture movement during continous and intermittent drying of Eucalyptus saligna, Eur J Wood Wood Prod., 2018, 76, 1165-1172.

15- A. El Brouzi, J.-M. Vergnaud, Diffusion of curing agent through the thickness of a bi-layer EPDM system during the cure, Polym Test., 2009, 28, 392-401.

16- M. Defo, A. Cloutier, Y. Fortin, Modeling vacuum-contact drying of wood: the water potential approach, Dry Technol., 2000, 18, 1737-1778.

17- W. Olek, J. Weres, Effects of the method of identification of the diffusion coefficient on accuracy of modeling bound water transfer in wood, In, Kowalski SJ (ed). Drying of Porous Materials, Springer Netherlands: Dordrecht, 2007, 135-144.

18- H. Mounji, M. El Kouali, J. M. Vergnaud, Process of Absorption of Moisture by Wood in Case of Condensation. Modelling and Experiment, J Polym Eng., 1993, 12, 197-218.

19- H. Mounji, J. Bouzon, J. M. Vergnaud, Modeling the process of absorption and desorption of water in two dimensions (transverse) in a square wood beam, Wood Sci Technol., 1991, 26.

20- A. Droin-Josserand, J. L. Taverdet, J. M. Vergnaud, Modelling of Moisture Absorption within a Section of Parallelepipedic Sample of Wood by Considering Longitudinal and Transversal Diffusion, Holzforschung, 1989, 43, 297-302.

21- W. Kang, W. Y. Chung, Liquid water diffusivity of wood from the capillary pressure-moisture relation, J Wood Sci., 2009, 55, 91-99.

22- A. Haloui, E. Kouali, J. Bouzon, J. M. Vergnaud, Process of absorption and desorption of methanol with a 3-dimensional transport through Picea wood, Wood Sci Technol., 1994, 28. 